\title{
Van der Waals-coupled electronic states in incommensurate double-walled carbon nanotubes
}

\author{
Kaihui Liu ${ }^{1 \dagger}$, Chenhao Jin ${ }^{1 \dagger}$, Xiaoping Hong ${ }^{1}$, Jihoon Kim ${ }^{1}$, Alex Zett ${ }^{1,2}$, Enge Wang ${ }^{3}$ and Feng Wang ${ }^{1,2 \star}$
}

\begin{abstract}
Non-commensurate two-dimensional materials such as a twisted graphene bilayer or graphene on boron nitride, consisting of components that have no finite common unit cell, exhibit emerging moiré physics such as novel Van Hove singularities ${ }^{1-3}$, Fermi velocity renormalization ${ }^{4,5}$, mini Dirac points ${ }^{6}$ and Hofstadter butterflies ${ }^{7-11}$. Here we use double-walled carbon nanotubes as a model system for probing moiré physics in incommensurate one-dimensional systems, by combining structural and optical characterizations. We show that electron wavefunctions between incommensurate innerand outer-wall nanotubes can hybridize strongly, contrary to the conventional wisdom of negligible electron hybridization due to destructive interference ${ }^{12,13}$. The chirality-dependent inter-tube electronic coupling is described by one-dimensional zone folding of the electronic structure of twisted-andstretched graphene bilayers. Our results demonstrate that incommensurate van der Waals interactions can be important for engineering the electronic structure and optical properties of one-dimensional materials.
\end{abstract}

Engineering and tailoring the properties of materials is of central importance in modern science and technology. In bulk materials this is realized mainly through modifying the strong covalent bond by changing the crystalline structure or doping chemical elements. However, in low-dimensional materials, electronic properties can also be engineered through modifying the weak non-covalent coupling, because the electron waves are not buried in the bulk and the inter-molecular electronic coupling becomes important. This is exemplified in artificially twisted bilayers of graphene/graphene and graphene/boron nitride, where new phenomena ranging from Van Hove singularities ${ }^{1-6}$ to Hofstadter butterflies ${ }^{7-11}$ emerge from van der Waals coupling between atomically thin two-dimensional (2D) layers. In this letter we investigate coupled electronic states in double-walled carbon nanotubes (DWNTs), a prototypical van der Waals-coupled one-dimensional (1D) material ${ }^{14-19}$, and reveal structure-dependent electron wave hybridization between incommensurate inner- and outer-wall carbon nanotubes.

DWNTs provide an ideal family of 1D structures to explore van der Waals electronic coupling because each DWNT is precisely defined at the atomic level and there are hundreds of different DWNT varieties. Specifically, a DWNT is uniquely characterized by the chiral indices $\left(n_{\mathrm{o}}, m_{\mathrm{o}}\right) /\left(n_{\mathrm{i}}, m_{\mathrm{i}}\right)$ of the constituent outer and inner single-walled carbon nanotubes (SWNTs), and a constituent SWNT can have a different electronic structure varying from metallic to semiconducting depending on its chiral index ${ }^{20}$. Electronic coupling between van der Waals-coupled DWNTs has long fascinated researchers ${ }^{21-26}$. Theoretical studies of commensurate DWNTs, where the inner and outer walls have commensurate carbon lattices and therefore are amenable to theoretical calculations, predict strong inter-tube coupling ${ }^{26}$. However, commensurate DWNTs have never been observed experimentally because it is almost impossible to have two commensurate SWNTs with the radius difference matching the tube-tube separation in a DWNT (refs 16-25). Electronic structure calculations of incommensurate DWNTs, on the other hand, are challenging because a finite unit cell does not exist. Several theoretical attempts studying incommensurate DWNTs suggest that inter-tube electronic coupling is negligible between the incommensurate inner- and outer-wall carbon lattices because couplings at different carbon atom sites oscillate with random phases and cancel each other ${ }^{12,13}$. Here we show experimentally that, contrary to previous theoretical predictions, electronic coupling from van der Waals interactions can be surprisingly strong in incommensurate DWNTs. The coupled electronic states can lead to either a blueshift or redshift of the optical transition energy by up to $150 \mathrm{meV}$, with the exact energy shift depending sensitively on the DWNT chirality. In addition, we develop a theory based on 1D zone folding of twisted-and-stretched graphene bilayers that successfully describes the coupled electronic states in incommensurate DWNT systems.

Inter-tube electronic coupling can be probed through the shift in optical transition energies of a DWNT compared with those from isolated constituent SWNTs. It requires accurate determination of the chiral structure and optical transition of individual DWNTs, which is achieved in our study by combining electron diffraction measurements ${ }^{18,19}$ and single-tube absorption spectroscopy ${ }^{27,28}$ on the same suspended DWNTs (Fig. 1a and Methods). Figure 1b,c shows, respectively, the electron diffraction pattern and absorption spectrum of a representative DWNT. The electron diffraction pattern (Fig. 1b) unambiguously determines the DWNT chiral indices $\left(n_{\mathrm{o}} m_{\mathrm{o}}\right) /\left(n_{\mathrm{i}} m_{\mathrm{i}}\right)$ to be $(22,9) /(11,11)$, which corresponds to a semiconducting outer-wall nanotube with a diameter of $2.16 \mathrm{~nm}$ and an armchair metallic inner-wall nanotube with a diameter of $1.49 \mathrm{~nm}$. Its absorption spectrum (Fig. 1c) shows four prominent optical resonances at 1.58, 1.66, 2.08 and $2.41 \mathrm{eV}$. In comparison, the isolated $(22,9)$ outer SWNT has three optical transitions $S_{33}^{o}$, $S_{44}^{\circ}$ and $S_{55}^{\circ}$ at $1.66,2.17$ and $2.58 \mathrm{eV}$, respectively, and the isolated $(11,11)$ inner SWNT has one $M_{11}^{\mathrm{i}}$ transition at $1.77 \mathrm{eV}$ in the experimental spectral range ${ }^{29}$. (Transition energies of the isolated SWNTs are indicated by vertical lines in Fig. 1c). There is a oneto-one correspondence between the DWNT optical resonances and those from the constituent SWNTs, but the resonance energies are shifted by $-80,-90,-170$ and $-110 \mathrm{meV}$ for the $S_{33}^{o}$, $S_{44}^{o}, S_{55}^{o}$ and $M_{11}^{\mathrm{i}}$ transitions, respectively. (The larger oscillator strength of the metallic $M_{11}^{\mathrm{i}}$ transition is because this transition

${ }^{1}$ Department of Physics, University of California at Berkeley, Berkeley, California 94720, USA, ${ }^{2}$ Materials Science Division, Lawrence Berkeley National Laboratory, Berkeley, California 94720, USA, ${ }^{3}$ International Center for Quantum Materials and Collaborative Innovation Center of Quantum Matter, Peking University, Beijing 100871, China. †These authors contributed equally to this work. *e-mail: fengwang76@berkeley.edu. 
a
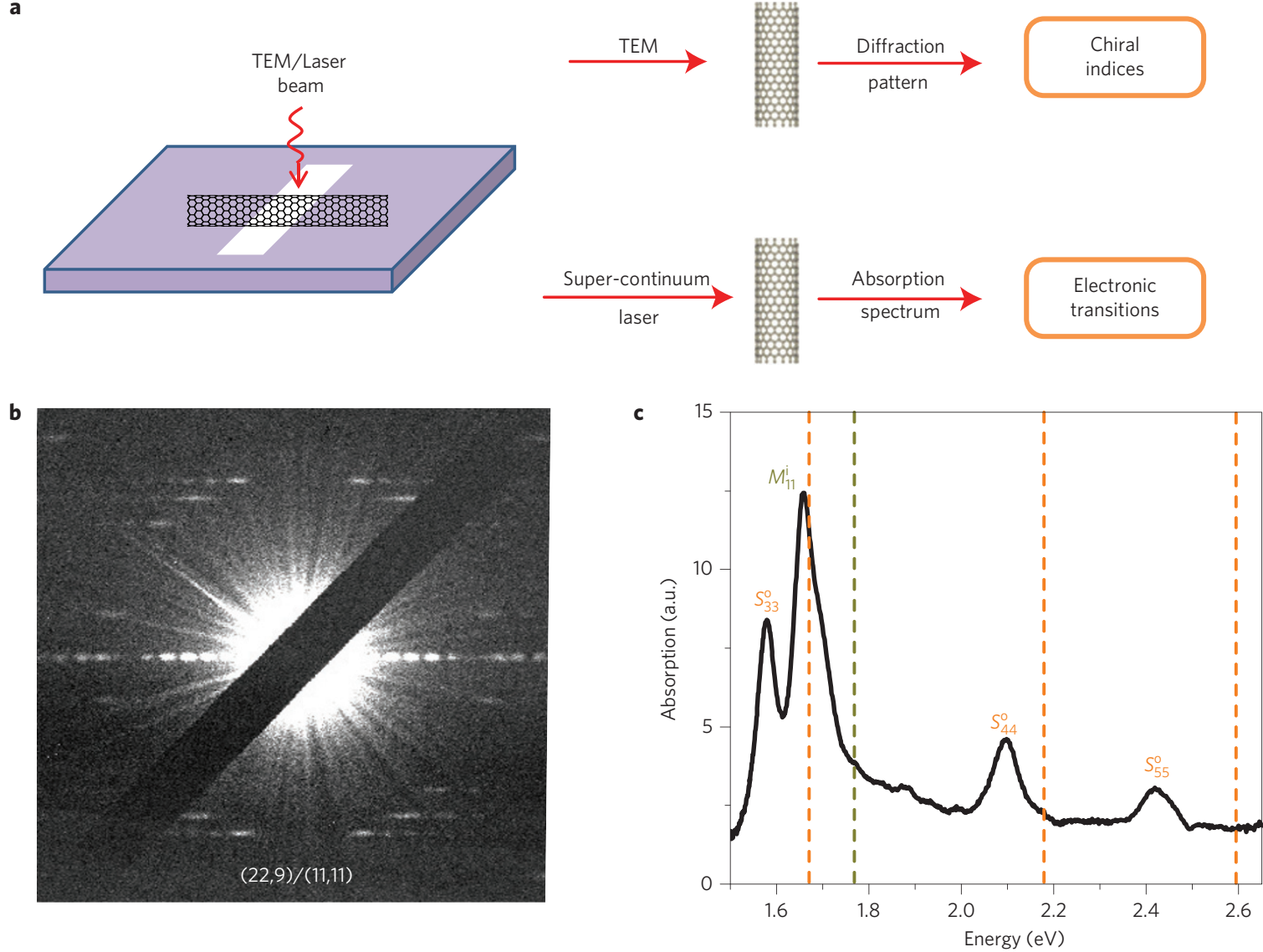

Figure 1 | Optical transitions of an individual chirality-defined DWNT. a, Schematic illustration of the experimental design. Chiral indices and optical transitions of the same individual suspended DWNT are obtained through single-tube transmission electron microscope (TEM) diffraction and absorption spectroscopy techniques, respectively. b. Experimental electron diffraction pattern of a $(22,9) /(11,11)$ DWNT. The outer (inner) tube is semiconducting (metallic) with a diameter of $2.16(1.49) \mathrm{nm}$. The horizontal zero-order and high-order diffraction bands in the diffraction pattern uniquely define the nanotube chiral index $(n, m)$. The black diagonal feature is from the blocking stick inside the TEM for dark-field imaging. $\mathbf{c}$, Absorption spectrum of the same DWNT, exhibiting strong optical resonances. A one-to-one correspondence exists between the DWNT optical transitions and those from the constituent SWNTs (dashed lines and Supplementary Fig. 1), but the resonance energies are shifted by $-80,-90,-170$ and -110 meV for the $S_{33}^{\circ}, S_{44}^{\circ}, S_{55}^{\circ}$ and $M_{11}^{i}$ transitions, respectively. This large transition energy shift demonstrates the strong inter-tube interactions between the incommensurate inner and outer tubes.

is doubly degenerate; see Supplementary Fig. 1 for more details). We performed combined electron diffraction and single-tube absorption measurements on 28 individual suspended DWNTs with a total of 99 optical transitions. In all studied DWNTs, we can map each observed optical transition to that from an isolated constituent SWNT, but the resonance energy is always shifted. (The optical transition energies in the SWNT are obtained from experiments or simulations $^{29}$, which have less than $20 \mathrm{meV}$ uncertainty.)

Figure 2 summarizes the observed resonance energy shifts for all optical transitions in DWNTs sorted by the shift value. All the DWNTs consist of incommensurate inner and outer walls, but the optical transition energy shift can be large, varying from a redshift of $190 \mathrm{meV}$ to a blueshift of $50 \mathrm{meV}$ in different DWNT species. Even for optical transitions from the same constituent SWNT, the energy shifts can vary significantly (Fig. 1c). Our observation signifies a very strong effect from inter-tube coupling in DWNTs, and this coupling depends sensitively on the exact electronic states in a DWNT.

In the literature the only well-established interaction between adjacent SWNTs is dielectric screening. It has been shown in previous studies of nanotubes in different environments ${ }^{30,31}$ that dielectric screening can lead to a redshift in optical transition energies, and the redshift value is similar for all optical transitions.
This dielectric screening effect can account for an average redshift of optical transition energies observed in DWNTs (Fig. 2), but it cannot explain the very large and strongly transition-dependent variations in optical transition shifts, especially the significant blueshifts for certain optical transitions. Obviously, other important intertube interactions are present in these incommensurate DWNTs, such as electron wavefunction hybridization between inner and outer walls.

To correctly describe the van der Waals-coupled electronic states in incommensurate DWNTs, we develop here a new perturbation theory based on 1D zone folding of twisted-and-stretched bilayer graphene. For an incommensurate DWNT, the supercell size is infinite owing to the irrational ratio between the inner- and outernanotube unit cell lengths. As a result, its electronic structure cannot be calculated using conventional tight-binding or ab initio methods based on periodic boundary conditions. In our calculation, we treat the interactions between electronic states from the constituent SWNTs perturbatively, and sum up all pair interactions between the inner and outer carbon lattices.

In a zone-folding scheme, electronic states of an isolated carbon nanotube are described by parallel cutting lines in the graphene Brillouin zone (GBZ) due to angular momentum quantization along the circumference. Therefore each SWNT electronic state can be 


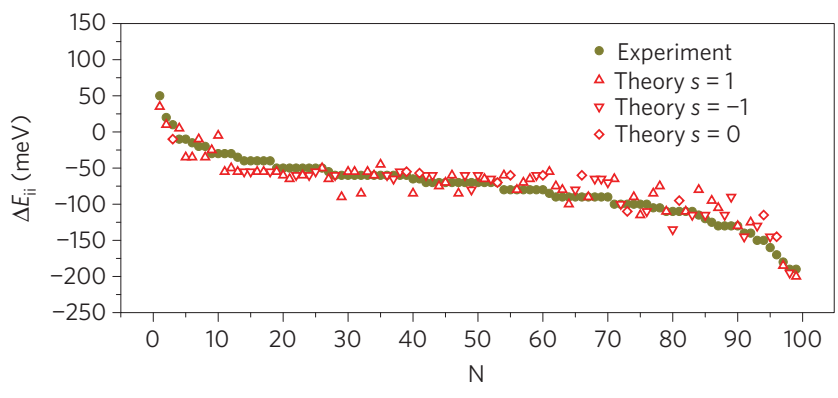

Figure 2 | Energy shifts of 99 transitions in 28 DWNTs compared with the corresponding constituent SWNT transitions. The optical transition energies in DWNTs are significantly shifted as a result of inter-tube interactions, ranging from $-200 \mathrm{meV}$ to $50 \mathrm{meV}$ in different DWNTs (circles). The average redshift $(\sim-55 \mathrm{meV})$ can be attributed to a dielectric screening effect. The large structure-dependent energy shift arises from the electronic coupling between the incommensurate inner and outer SWNTs. Our theory, including the inter-tube electronic coupling and the dielectric screening (diamonds and triangles), successfully reproduces the experimental data (circles). Up triangles, down triangles and diamonds represent transitions from DWNTs with $s=1$ (two walls of the same handedness), -1 (two walls of opposite handedness) and 0 (at least one wall is armchair or zigzag), respectively.

characterized by $\mu$, the quantized angular wavevector indexing a cutting line; $k$, the continuous linear wavevector along the cutting line; and $\sigma$, indexing the valence/conduction band $^{20}$. In a DWNT, the electronic coupling between an inner-tube state $\alpha(\mu, k, \sigma)$ and an outer-tube state $\beta\left(\mu^{\prime}, k^{\prime}, \sigma^{\prime}\right)$ is determined by summing up all the carbon-carbon pair interactions between the inner and outer SWNTs. The coupling matrix element can be expressed as (Supplementary Information, Section 2):

$$
M_{\alpha \beta}=\frac{1}{2 \sqrt{N N^{\prime}}} \sum_{\mathbf{R}_{j}} \mathrm{e}^{i\left[\left(\mu^{\prime}-\mu\right) \theta_{j}+\left(k^{\prime}-k\right) z_{j}\right]} \sum_{\mathbf{R}_{i}^{\prime}} \mathrm{e}^{i\left[\mu^{\prime} \Delta \theta+k^{\prime} \Delta z\right]} t_{\sigma \sigma^{\prime}}(\Delta \theta, \Delta z)
$$

Here $N\left(N^{\prime}\right)$ is the total number of graphene unit cells on the inner (outer) nanotube; $\mathbf{R}_{j}=\left(\theta_{j}, z_{j}\right)$ is the coordinate of an inner-tube unit cell and $\Delta \mathbf{R}=\mathbf{R}_{i}^{\prime}-\mathbf{R}_{j}=(\Delta \theta, \Delta z)$ is the displacement of an outer-tube unit cell at $\mathbf{R}_{i}^{\prime}$ relative to $\mathbf{R}_{j} ; t_{\sigma \sigma^{\prime}}(\Delta \theta, \Delta z)$ is the coupling coefficient between two interlayer unit cells. The first (second) summation goes over inner-tube (outer-tube) lattices. It is clear that the summed terms have an oscillating phase changing with carbon atom positions. This oscillating phase leads to the commonly held belief that overall electronic coupling in incommensurate DWNTs will average to zero ${ }^{12,13}$. Although this is mostly true for random pairs of electronic states, there are important exceptions: an outertube state $\beta\left(\mu^{\prime}, k^{\prime}, \sigma^{\prime}\right)$ can have finite electronic coupling to an innertube state $\alpha(\mu, k, \sigma)$ if it satisfies the requirement:

$$
\begin{gathered}
q^{\prime}=q+G_{i}, \\
q=\frac{\mu}{r_{\mathrm{i}}} \theta+k z, q^{\prime}=\frac{\mu}{r_{\mathrm{o}}} \frac{r_{\mathrm{o}}}{r_{\mathrm{i}}} \theta+k^{\prime} z=\eta \frac{\mu}{r_{\mathrm{o}}} \theta+k^{\prime} z
\end{gathered}
$$

where $r_{\mathrm{i}}\left(r_{\mathrm{o}}\right)$ is the inner-tube (outer-tube) radius, $\theta(z)$ is the circumferential (axial) unit vector and $G_{i}$ is the reciprocal lattice vector of the inner tube's 2D GBZ (Supplementary Information, Section 3). Here $q$ is the wavevector in the 2D GBZ defining the inner-tube state $\alpha$, and $q^{\prime}$ is the wavevector in the 'stretched' $2 \mathrm{D}$ Brillouin zone (by $\eta=r_{\mathrm{o}} / r_{\mathrm{i}}$ along the circumferential direction) of a 'compressed' graphene defining the outer-tube state $\beta$. This effective 'compression' is a unique characteristic of 1D DWNTs, which makes the originally different inner- and outer-tube cutting line spacing (Fig. 3a) the same. Figure $3 \mathrm{~b}$ shows the 2D GBZs associated with the inner (pink hexagons) and outer tubes (grey hexagons), where the effective 'compression' of the outer nanotube leads to a 'stretching' of the corresponding GBZ. In this scheme, the available inner- and outer-nanotube states (with quantized angular momentum) lie on the same parallel cutting lines. In Fig. $3 \mathrm{~b}$ the requirement of equation (1) has a clear physical picture: an outer-tube state $\beta$ can couple to the inner-tube state $\alpha$ only if its corresponding $2 \mathrm{D}$ wavevector $q^{\prime}$ matches $q+G_{i}$, wavevectors of states equivalent to $\alpha$ in the extended GBZ of the inner-tube ( $q$ and $q^{\prime}$ overlapped at filled circles in Fig. 3b).

For coupled inner- and outer-tube electronic states satisfying the condition of equation (1), the coupling matrix element has the form:

$$
M=C \delta_{q+G_{i}, q^{\prime}} \mathrm{e}^{-(\lambda \Delta r / 2)\left(k^{\prime 2}+\mu^{\prime 2} / r_{\mathrm{i}} r_{\mathrm{o}}\right)}
$$

where $C$ and $\lambda$ are parameters. The value of $M$ can be calculated quantitatively for all coupled states (Supplementary Information, Section 3), and its magnitude decays exponentially with $q^{\prime}=\left|q+G_{i}\right|$. Therefore only electronic states closest to the $\Gamma$ point (red filled circles in Fig. 3b) are important, and effects from other states are more than 100 times smaller. We evaluated the coupling matrix elements for electronic states corresponding to all optical transitions observed in our DWNTs, where the twisting angle between the inner and outer walls varies from $0.5^{\circ}$ to $25^{\circ}$ and the inter-tube distance varies from 0.33 to $0.38 \mathrm{~nm}$. We found that the largest coupling between electronic states $\left(M_{\max }\right)$ does not have a simple relation with the inter-tube twisting angle. However, the coupling strength depends very sensitively on the inter-tube separation (diamonds in Fig. 3c), which can be described by an exponential decay (red line in Fig. 3c).

With knowledge of the matrix elements, we can now quantitatively estimate the transition energy shifts induced by the inter-tube electronic coupling using perturbation theory with (see Supplementary Information, Section 3)

$$
\delta E_{\mathrm{ii}}^{\mathrm{el}}=\sum_{\beta=1}^{3} \frac{\left|A_{\mathrm{i} \beta}\right|^{2}}{E_{\mathrm{i}}-E_{\beta}}=\sum_{\beta=1}^{3} \frac{\left|A_{\mathrm{i} \beta}\right|^{2}}{\Delta E_{\mathrm{i} \beta}}
$$

Here $\delta E_{\mathrm{ii}}^{\mathrm{el}}$ is the transition energy shift of a specific inner-tube (outertube) optical transition $E_{\mathrm{ii}}$, and $M_{\mathrm{i} \beta}$ and $\Delta E_{\mathrm{i} \beta}$ are, respectively, the coupling matrix element and energy difference between the innertube (outer-tube) state $\mathrm{i}$ and outer-tube (inner-tube) state $\beta$. It is obvious that the $1 / \Delta E_{\mathrm{i} \beta}$ is critically important in determining the final energy shift, and its value varies systematically on multiple parameters, including the inner-wall diameter and chiral angle, the outer-wall diameter and chiral angle, and the specific optical transitions. As an example, we examine the maximum value of $1 / \Delta E_{\mathrm{i} \beta}$ for the inner-tube $S_{22}^{\mathrm{i}}$ transitions with diameter $d=1 \mathrm{~nm}$, and plot its dependence on the inner-tube chiral angle $\left(\theta_{\mathrm{i}}\right)$ and the inter-tube twisting angle $\left(\theta_{\mathrm{twist}}\right)$ in Fig. 3d,e. The figure shows distinct behaviour for inner semiconducting tubes of the $\bmod (n-m, 3)=1$ family (Fig. $3 \mathrm{~d})$ and the $\bmod (n-m, 3)=2$ family (Fig. 3e). In addition, a large value of $\left|1 / \Delta E_{\mathrm{i} \beta}\right|$, corresponding to the white region in the figures, occurs only when the twist angle $\theta_{\text {twist }}$ satisfies a specific relation with regard to $\theta_{\mathrm{i}}$. Similar systematic behaviour of $1 / \Delta E_{\mathrm{i} \beta}$ can be obtained for different optical transitions and for DWNTs of different diameters and inner- and outer-wall chiral angles (see Supplementary Information, Section 4 more details).

Combining the information on $M_{\mathrm{i} \beta}$ and $\Delta E_{\mathrm{i} \beta}$, we can predict optical transition energy shifts $\delta E_{\mathrm{ii}}^{\mathrm{el}}$ due to inter-tube electronic 


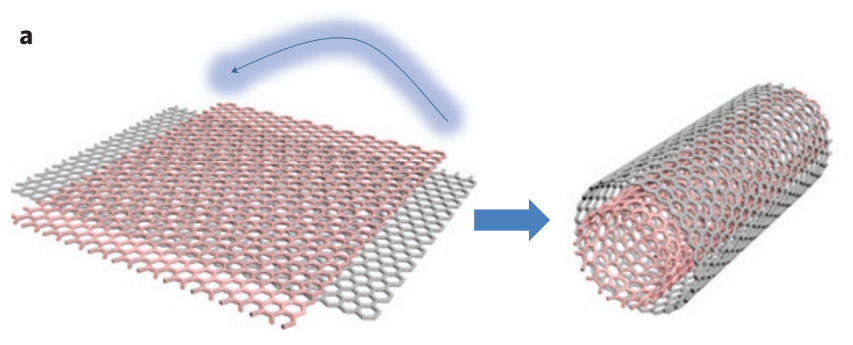

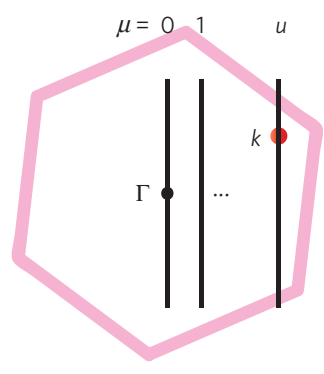

C

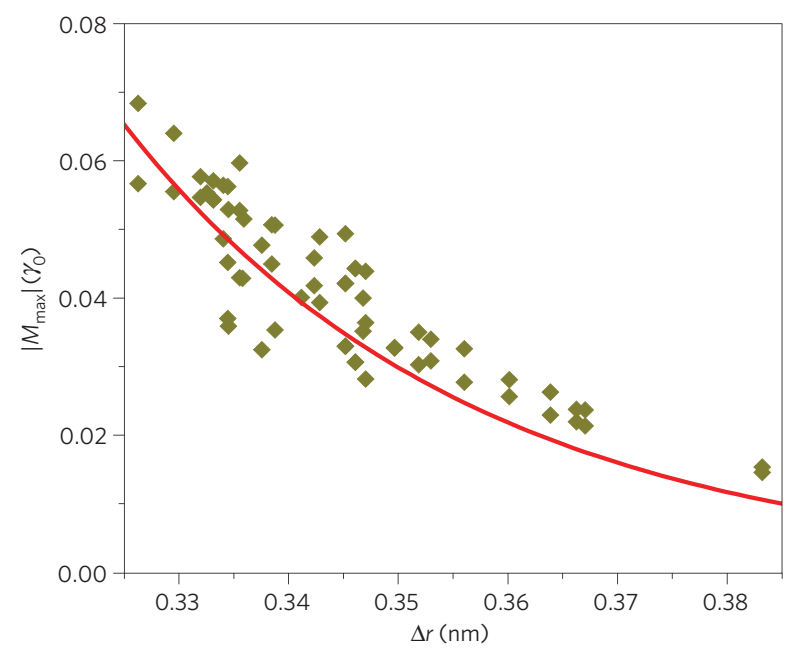

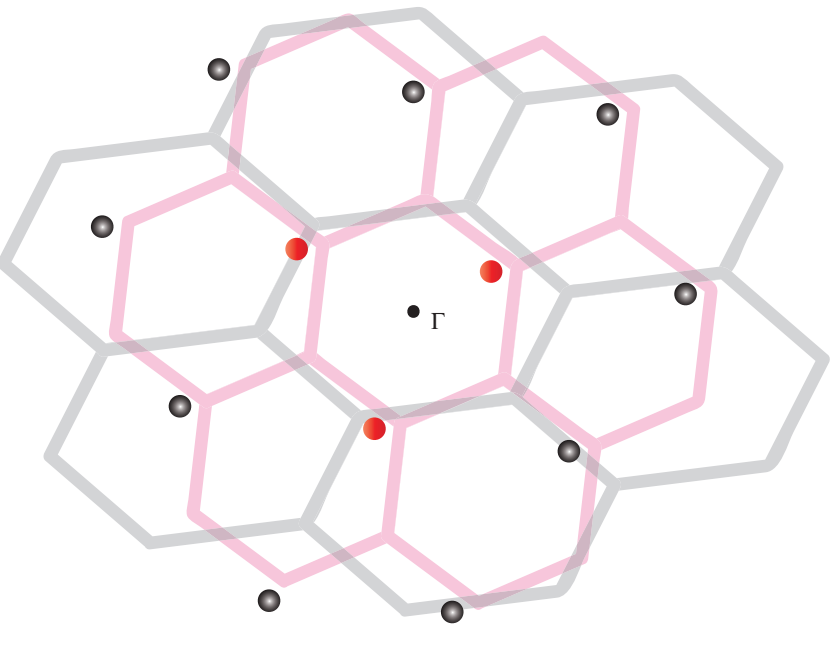

d

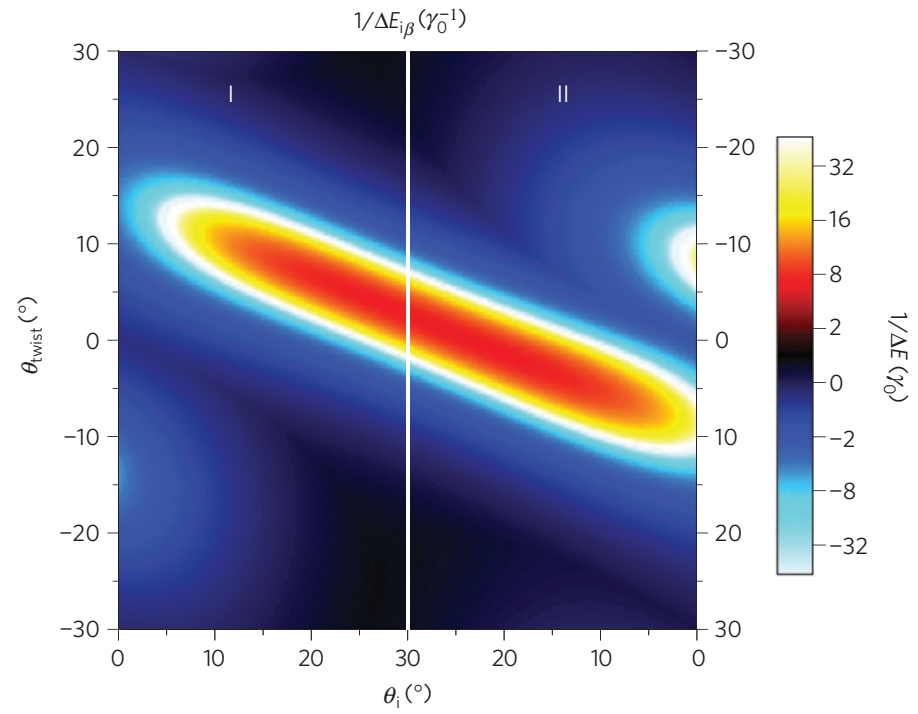

Figure 3 | Chirality dependence of inter-tube electronic coupling in an incommensurate DWNT. a, Zone folding picture of the electronic states in the 2D graphene Brillouin zone (GBZ) for the inner SWNT (pink colour) and the outer SWNT (grey colour). The inner and outer tubes have different radii in real space (upper panel) and therefore a different cutting line spacing in momentum space (lower panel). The orientation of the 2D GBZ is determined by the SWNT chiral angle with cutting lines fixed along the $y$ (axial)-direction. $\mathbf{b}$, The requirement of finite inter-tube electronic coupling illustrated in the extended 2D GBZ. The outer-tube GBZ (grey) is stretched by $r_{0} / r_{i}$ and placed on the inner-tube GBZ (pink), so that the inner- and outer-tube electronic states are described by the same cutting lines in the 2D extended GBZ. For an inner-tube electronic state at wavevector $q$, it can only couple to outer-tube

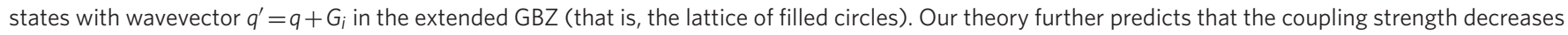
exponentially with increasing $q^{\prime}$. Consequently, only three outer-wall states close to the $\Gamma$ point (labelled with red filled circles) can couple strongly with the inner-tube electronic state at $q$. c, Largest coupling of an electronic state $\left(\left|M_{\max }\right|\right)$ for our studied optical transitions in DWNTs as a function of the inter-tube separation $\Delta r$, which shows a strong exponential dependence (red line). $\gamma_{0}=3 \mathrm{eV}$. d,e, Dependence of $1 / \Delta E_{\mathrm{i} \beta}$ on the inner-tube chiral angle $\theta_{\mathrm{i}}$ and inter-tube twist angle $\theta_{\text {twist }}$ for the inner-tube $S_{22}^{i}$ transition in DWNTs with $d_{i}=1.0 \mathrm{~nm}$ and $d_{0}=1.7 \mathrm{~nm}$. A small $\Delta E_{i} \beta$, the energy difference between the coupled electronic states, is critical for a large coupling-induced energy shift. The value of $1 / \Delta E_{i} \beta$ has a systematic dependence on the nanotube chiral angles and exhibits a distinct family pattern for the $\bmod (n-m, 3)=1$ family $(\mathbf{d})$ and the $\bmod (n-m, 3)=2$ family $(\mathbf{e})$.

coupling in all DWNTs. For example, Figure 4 illustrates the optical transition shifts of a $(15,10)$ inner-wall tube when it is coupled to different outer-wall tubes of chirality $(24,11),(20,15)$ and $(18,17)$, respectively. It is obvious that, for the same inner tube, the energy shift of an optical transition $\delta E_{\mathrm{ii}}^{\mathrm{el}}$ varies strongly with the outer-wall tube species, where the shift can be either positive or negative and has a magnitude as large as $150 \mathrm{meV}$.

In addition to the electronic coupling discussed above, dielectric screening between the inner and outer tubes also contributes to the shift of optical transition energies. For simplicity we assume that dielectric screening leads to a constant redshift $\Delta$ for all transitions, and use it as the only fitting parameter to describe the experimental data. Therefore, the total energy shift $\delta E_{\mathrm{ii}}^{\text {tot }}=\delta E_{\mathrm{ii}}^{\mathrm{el}}+\Delta$. Figure 2 shows the comparison between the theoretical prediction (triangles and diamonds) and experimental data (circles) for all optical transitions probed in our study using $\Delta=-55 \mathrm{meV}$. We note that the handedness $(n>m$ or $n<m)$ does not affect the optical spectra of SWNTs; however, in DWNTs the relative handedness between constituent tubes can affect the inter-tube electronic coupling strength (except for the case in which the DWNT contains at least 


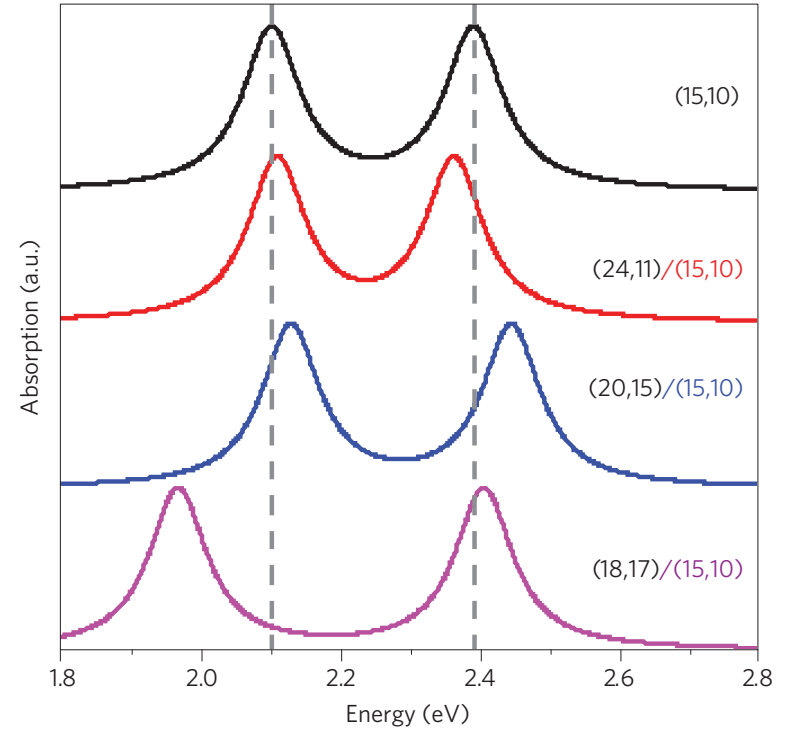

Figure 4 | Simulation of the inter-tube electronic coupling-induced optical transition shift for a $(15,10)$ inner SWNT. Optical resonances $\left(S_{33}\right.$ and $\left.S_{44}\right)$ from the $(15,10)$ nanotube show a significant energy shift due to the inter-tube coupling. The amplitude of energy shift depends sensitively on the specific optical transition and the outer-tube species, which can be either positive or negative, and has a magnitude as large as $150 \mathrm{meV}$.

one armchair or zigzag tube). Here we introduce an 's' parameter indicating whether the inner and outer tubes have the same $(s=1)$ or opposite $(s=-1)$ handedness. The electron diffraction pattern cannot determine the relative handedness (that is, the $s$ parameter) experimentally. Instead we assign the $s$ parameter based on a better match between the experimental and theoretical transition energy shifts. In Fig. 2 up triangles, down triangles and diamonds represent transitions in DWNTs with $s=1,-1$ and 0 (contains armchair or zigzag tube), respectively. The agreement between experimental and theoretical results is fairly good given that all parameters in the electronic coupling model are obtained from literature values and the energy shift from the dielectric screening $\Delta$ is the only fitting parameter. The residual deviation might be due to a dielectricscreening-induced redshift different from the assumed $-55 \mathrm{meV}$ and the uncertainty in experimental data $(\sim 20 \mathrm{meV})$. Detailed information of all DWNT optical transitions and their comparison with the theoretical predictions can be found in Supplementary Table 1.

The inter-tube electronic coupling observed here is fundamental for understanding the physical properties of double-walled and multi-walled carbon nanotubes. More generally, our study shows that van der Waals coupling can strongly modify the electronic structures of $1 \mathrm{D}$ as well as $2 \mathrm{D}$ incommensurate systems. It opens up new avenues in engineering the electronic and optical properties of van der Waals-coupled low-dimensional materials.

\section{Methods}

In our experiment we use suspended DWNTs that are free of substrate effects and compatible with both transmission electron microscope (TEM) diffraction and single-tube optical spectroscopic techniques. Long suspended nanotubes were grown by chemical vapour deposition across open slit structures $(\sim 30 \times 500 \mu \mathrm{m})$ fabricated on silicon substrates. We use iron nanoparticles as catalysts and methane in hydrogen $\left(\mathrm{CH}_{4}: \mathrm{H}_{2}=1: 2\right)$ as gas feedstock, and control the size of the catalyst particles to achieve the selective abundance of DWNTs (ref. 32). We determine the chiral structures of DWNTs using electron diffraction with nano-focused $80 \mathrm{keV}$ electron beams in a JEOL 2100 TEM (refs 18,19). Using the slit edges as markers, the same individual nanotubes can be identified in an optical microscopy set-up. To obtain the absorption spectra of these individual nanotubes, we use polarization-based transmission microscopy with a supercontinuum light source covering the spectral range from 450 to $1,600 \mathrm{~nm}$ (refs 27,28).

Received 6 December 2013; accepted 24 June 2014; published online 7 September 2014

\section{References}

1. Li, G. H. et al. Observation of Van Hove singularities in twisted graphene layers. Nature Phys. 6, 109-113 (2010).

2. Kim, K. et al. Raman spectroscopy study of rotated double-layer graphene: Misorientation-angle dependence of electronic structure. Phys. Rev. Lett. 108, 246103 (2012)

3. Havener, R. W. et al. Angle-resolved Raman imaging of interlayer rotations and interactions in twisted bilayer graphene. Nano Lett. 12, 3162-3167 (2012).

4. De Laissardiere, G. T., Mayou, D. \& Magaud, L. Localization of Dirac electrons in rotated graphene bilayers. Nano Lett. 10, 804-808 (2010).

5. Luican, A. et al. Single-layer behavior and its breakdown in twisted graphene layers. Phys. Rev. Lett. 106, 126802 (2011).

6. Yankowitz, M. et al. Emergence of superlattice Dirac points in graphene on hexagonal boron nitride. Nature Phys. 8, 382-386 (2012).

7. Hofstadter, D. R. Energy levels and wave functions of Bloch electrons in rational and irrational magnetic fields. Phys. Rev. B 14, 2239-2249 (1976).

8. Bistritzer, R. \& MacDonald, A. H. Moire bands in twisted double-layer graphene. Proc. Natl Acad. Sci. USA 108, 12233-12237 (2011).

9. Dean, C. R. et al. Hofstadter's butterfly and the fractal quantum Hall effect in moire superlattices. Nature 497, 598-602 (2013).

10. Hunt, B. et al. Massive Dirac fermions and Hofstadter butterfly in a van der Waals heterostructure. Science 340, 1427-1430 (2013).

11. Ponomarenko, L. A. et al. Cloning of Dirac fermions in graphene superlattices. Nature 497, 594-597 (2013).

12. Frank, S., Poncharal, P., Wang, Z. L. \& de Heer, W. A. Carbon nanotube quantum resistors. Science 280, 1744-1746 (1998).

13. Uryu, S. \& Ando, T. Electronic intertube transfer in double-wall carbon nanotubes. Phys. Rev. B 72, 245403 (2005).

14. Saito, R. et al. Anomalous potential barrier of double-wall carbon nanotube. Chem. Phys. Lett. 348, 187-193 (2001).

15. Bandow, S. et al. Raman scattering study of double-wall carbon nanotubes derived from the chains of fullerenes in single-wall carbon nanotubes. Chem. Phys. Lett. 337, 48-54 (2001).

16. Zuo, J. M. et al. Atomic resolution imaging of a carbon nanotube from diffraction intensities. Science 300, 1419-1421 (2003).

17. Hashimoto, A. et al. Atomic correlation between adjacent graphene layers in double-wall carbon nanotubes. Phys. Rev. Lett. 94, 045504 (2005).

18. Hirahara, K. et al. Chirality correlation in double-wall carbon nanotubes as studied by electron diffraction. Phys. Rev. B 73, 195420 (2006).

19. Liu, K. H. et al. Direct determination of atomic structure of large-indexed carbon nanotubes by electron diffraction: Application to double-walled nanotubes. J. Phys. D 42, 125412 (2009).

20. Saito, R., Dresselhaus, M. S. \& Dresselhaus, G. Physical Properties of Carbon Nanotubes (World Scientific Publishing Company, 1998).

21. Kociak, M. et al. Linking chiral indices and transport properties of double-walled carbon nanotubes. Phys. Rev. Lett. 89, 155501 (2002).

22. Villalpando-Paez, F. et al. Raman spectroscopy study of isolated double-walled carbon nanotubes with different metallic and semiconducting configurations. Nano Lett. 8, 3879-3886 (2008).

23. Liu, K. H. et al. Chirality-dependent transport properties of double-walled nanotubes measured in situ on their field-effect transistors. J. Am. Chem. Soc. 131, 62-63 (2009).

24. Levshov, D. et al. Experimental evidence of a mechanical coupling between layers in an individual double-walled carbon nanotube. Nano Lett. 11, 4800-4804 (2011).

25. Liu, K. H. et al. Quantum-coupled radial-breathing oscillations in double-walled carbon nanotubes. Nature Commun. 4, 1375 (2013).

26. Moradian, R., Azadi, S. \& Refii-Tabar, H. When double-wall carbon nanotubes can become metallic or semiconducting. J. Phys. Condens. Matter 19, 176209 (2007)

27. Lefebvre, J. \& Finnie, P. Polarized light microscopy and spectroscopy of individual single-walled carbon nanotubes. Nano Res. 4, 788-794 (2011).

28. Liu, K. H. et al. High-throughput optical imaging and spectroscopy of individual carbon nanotubes in devices. Nature Nanotech. 8, 917-922 (2013).

29. Liu, K. et al. An atlas of carbon nanotube optical transitions. Nature Nanotech. 7, 325-329 (2012) 
30. Lefebvre, J., Fraser, J. M., Homma, Y. \& Finnie, P. Photoluminescence from single-walled carbon nanotubes: A comparison between suspended and micelle-encapsulated nanotubes. Appl. Phys. A 78, 1107-1110 (2004)

31. Steiner, M. et al. How does the substrate affect the Raman and excited state spectra of a carbon nanotube? Appl. Phys. A 96, 271-282 (2009).

32. Huang, S. M., Cai, X. Y. \& Liu, J. Growth of millimeter-long and horizontally aligned single-walled carbon nanotubes on flat substrates. J. Am. Chem. Soc. 125, 5636-5637 (2003)

\section{Acknowledgements}

This study was supported mainly by NSF grants (No. DMR-0846648 and DMR-1404865) and the NSF Center for Integrated Nanomechanical Systems (No. EEC-0832819). Support for the TEM characterization and sample preparation was provided by the

Director, Office of Energy Research, Materials Sciences and Engineering Division of the US Department of Energy under Contract No. DE-AC02- 05CH11231.

\section{Author contributions}

F.W., K.L. and C.J. conceived the project. K.L., J.K. and A.L. contributed to sample growth and characterization. K.L. carried out TEM measurements. K.L. and X.H. carried out optical measurements. K.L. and C.J. analysed the experimental data. C.J., E.W. and F.W. developed the theory. K.L., C.J. and F.W. wrote the manuscript. All authors discussed the results and commented on the paper.

\section{Additional information}

Supplementary information is available in the online version of the paper. Reprints and permissions information is available online at www.nature.com/reprints.

Correspondence and requests for materials should be addressed to F.W.

\section{Competing financial interests}

The authors declare no competing financial interests. 